\title{
Heavy-Atom-Free Room-Temperature Phosphorescent Organic Light-Emitting Diodes Enabled by Excited States Engineering
}

\author{
Heather F. Higginbotham ${ }^{\dagger}$ Masato Okazaki, ${ }^{\ddagger}$ Piotr de Silva, ${ }^{*} \S$ Satoshi Minakata, ${ }^{\ddagger}$ Youhei Takeda,*,* \\ and Przemyslaw Data*,\|,L,\# \\ ${ }^{\dagger}$ School of Science, Swinburne University of Technology, Hawthorne, Victoria, Australia. \\ *Department of Applied Chemistry, Graduate School of Engineering, Osaka University, Yamadaoka 2-1, Suita, Osaka 565- \\ 0871, Japan \\ ${ }^{\S}$ Department of Energy Conversion and Storage, Technical University of Denmark, Anker Engelunds Vej 301, 2800 Kgs. \\ Lyngby, Denmark. \\ "Faculty of Chemistry, Silesian University of Technology, M. Strzody 9, 44-100 Gliwice, Poland \\ ${ }^{\perp}$ Physics Department, Durham University, South Road, Durham DH1 3LE, United Kingdom \\ ${ }^{\#}$ Centre of Polymer and Carbon Materials, Polish Academy of Sciences, M. Curie-Sklodowskiej 34, 41-819 Zabrze, Poland
}

\begin{abstract}
Room temperature phosphorescence materials offer great opportunities for applications in optoelectronics, due to their unique photophysical characteristics. However, purely organic emitters that can realize distinct electrophosphorescence are rarely exploited. Herein a new approach for designing heavy-atom-free organic room temperature phosphorescence emitters for organic light-emitting diodes is presented. The subtle tuning of the energy diagrams of singlet and triplet excited states by appropriate choice of host matrix allows tailored emission properties and switching of emission channels between thermally activated delayed fluorescence and room temperature phosphorescence. Moreover, an efficient and heavy-atom-free room temperature phosphorescence organic light-emitting diodes using the developed emitter is realized.
\end{abstract}

\section{Introduction}

Electroluminescence of organic molecules is one of the groundbreaking discoveries of the last century. ${ }^{1}$ Owing to it and the significant advancement in organic electronics, we are surrounded by organic light-emitting diode (OLED) products. ${ }^{2,3}$ In addition to lightness, flexibility, and low production cost, the major advantage of using organic emitters is that their properties are tailored by modifying their molecular structures. ${ }^{3-5}$ From the viewpoint of emission mechanisms, there are two types of emitters: fluorescence and phosphorescence emitters. Fluorescence emitters are known as the $1^{\text {st }}$ generation emitters for OLEDs, but they fatally have a problem of a discouraging upper limit (25\%) of internal quantum efficiency (IQE), because of the spin-statistics issue. The $2^{\text {nd }}$ generation emitters, phosphorescence emitters, can show much higher IQE up to $100 \%$, and for this reason, they are commonly used in OLEDs displays. ${ }^{2,3,6,7}$ However, currently used phosphorescence emitters are organometallic complexes that contain expensive and rare heavy metals. There are two approaches to achieve $100 \%$ IQE in OLED devices with metal-free emitters: the utilization of purely organic 1) thermally activated delayed fluorescence (TADF) emitters ${ }^{8-11,14}$ or 2) room temperature phosphorescence (RTP) emitters. ${ }^{12-14}$ TADF emitters, which are dubbed as $3^{\text {rd }}$ generation emitters, can harvest $100 \%$ of electrically-generated excitons by converting triplet states $\left(\mathrm{T}_{1}\right)$ into energetically-close singlet excited states $\left(\mathrm{S}_{1}\right)$ with thermal energies and thereby can emit photons through irradiative relaxation to the ground state $\left(\mathrm{S}_{0}\right)$. An issue of TADF emitters is a broad Gaussian-type emission spectrum, which might be suitable for lighting applications but not for the biggest market, displays. ${ }^{15,16}$ Although purely organic and Heavy-Atom-Free (HAF) RTP emitters would be a promising alternative as OLEDs emitters, this class of emitters has been rarely exploited. For example, Chaudhuri et al. reported that RTP can contribute to the emission from an OLED fabricated with small organic $\pi$-conjugated compounds with light atoms $(\mathrm{C}, \mathrm{H}$, and $\mathrm{N}),{ }^{13}$ but external quantum efficiencies (EQEs) of the devices were technically too low $\left(<10^{-4} \%\right)$.

From a mechanistic point of view, TADF and RTP processes appear to be opposing ones (from $T_{1}$ to $S_{1}$ followed by $S_{1}$ to $S_{0}$, and from $T_{1}$ to $S_{0}$ transition, respectively). But, theoretically, it should be possible to connect them, as both processes are required to have strong spin-orbit coupling (SOC) to increases efficiency. To endow emissive organic compounds with RTP characteristics, molecular rotations and vibrations should be locked so that non-irradiative deactivation pathways are shutout. ${ }^{17}$ On the other hand, to realize TADF characteristics, vibrations between electron-donor (D) and the acceptor (A) moieties are necessary. ${ }^{17,18}$ As we presented before, it is possible to slow down the rotation between donor and acceptor moiety in order to observe the RTP process by attaching bulky, steric hindering groups. ${ }^{19}$ The limiting value here is the temperature and decay lifetime. Phosphorescence is efficient at low temperature. On the other hand, by increasing temperature, we observe reverse intersystem crossing (RISC) and thereby TADF occurs, but the phosphorescence is not observed. In order to hinder the possibility of the TADF process, the singlet-triplet gap $\left(\Delta E_{\mathrm{ST}}\right)$ needs to be theoretically higher than $0.4 \mathrm{eV}$. Nevertheless, in spite of these conflicting design criteria, it is possible to switch between RTP and TADF processes by choosing appropriate hosts and control the $\Delta E_{\mathrm{ST} .}{ }^{19-22}$ Generally, TADF occurs 
through the RISC from an excited local triplet state $\left({ }^{3} \mathrm{LE}\right)$ to the excited charge transfer singlet state $\left({ }^{1} \mathrm{CT}\right)$, with the ${ }^{1} \mathrm{CT}$ energy highly sensitive toward host polarity (Figure 1a). Therefore, if the $\Delta E_{\mathrm{ST}}$ lies on the edge of the TADF process, it would be feasible to boost the RTP emission in a certain temperature range (Figure 1b). Nevertheless, an overlooked issue regarding purely organic RTP compounds is that the irradiative deactivation pathway from the $T_{1}$ state is commonly a very slow process (typical lifetime $>1 \mathrm{~ms}$ ). This disproves the possibility of applying such RTP-active organic compounds for OLED application, as the efficiency and stability of those OLEDs are poor, mainly due to exciton-polaron quenching and triplet-triplet annihilation (TTA). To overcome these issues, we decided to utilize a faster phosphorescence pathway from the $\mathrm{T}_{2}$ state which are accessible through thermally activated reverse internal conversion (RIC) process from longer-lived $\mathrm{T}_{1}$ state, where the $\mathrm{S}_{1}\left({ }^{1} \mathrm{CT}_{1}\right)$ is higher-lying than $T_{2}$ and $T_{1}$ states (Figure 1c). According to literature, the CT character of $\mathrm{T}_{2}$ state and a large SOC between $\mathrm{T}_{2}$ and $\mathrm{S}_{0}$ states should result in the much faster irradiative transition from $T_{2}$ than that from $T_{1}$ state to suppress the triplet quenching. ${ }^{24}$

Herein, we present a novel TADF/RTP hybrid HAF emitter SiAz (Figure 1c) comprising of naturally-abundant elements only $(\mathrm{C}, \mathrm{H}, \mathrm{N}$, and $\mathrm{Si})$. In addition to the material design, our theoretical investigation gives proof that the excited states energy alignment $\left(S_{1}, T_{1}\right.$, and $\left.T_{2}\right)$ of the emitter is engineered by host materials by affecting CT energy levels with polarity effect and inverting the energy levels of ${ }^{3} \mathrm{CT}$ and ${ }^{3} \mathrm{LE}$ states, leading to switching TADF and RTP outcomes. Most importantly, the developed emitter can be used to a HAF organic electrophosphorescent device with the highest EQE reported so far.
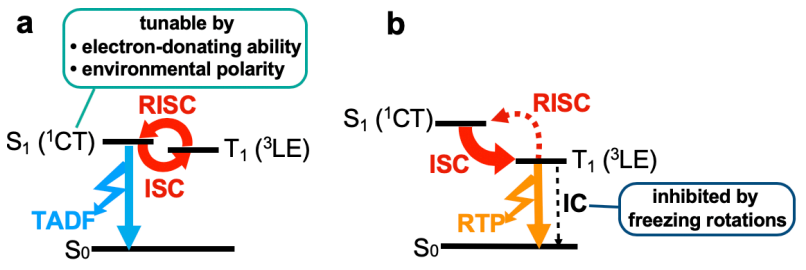

c
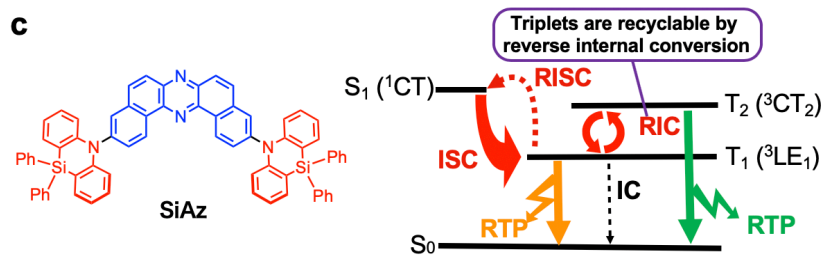

Figure 1. (a) The schematic Jablonski diagram for typical TADF emitters. (b) The schematic Jablonski diagram for previously studied RTP emitters. (c) The schematic Jablonski diagram for designed emitter (SiAz).

\section{Results and Discussions \\ Design and Synthesis of SiAz.}

To realize the HAF-RTP emitter that satisfies the abovementioned conflicting requisites, we designed a donor-acceptordonor (D-A-D) molecule SiAz (Figure 1c) comprising of naturally-abundant elements only $(\mathrm{C}, \mathrm{H}, \mathrm{N}$, and $\mathrm{Si})$. The design principles for $\mathbf{S i A z}$ involve the installation of i) weaker electron-donors (i.e., dihydrophenazasiline) than phenoxazine ${ }^{25}$ and phenothiazine ${ }^{23}$ to increase the $\Delta E_{\mathrm{ST}}$ by destabilizing the ${ }^{1} \mathrm{CT}$ state so that RISC becomes less effective and to make $T_{2}$ state below $\mathrm{S}_{1}\left({ }^{1} \mathrm{CT}\right)$ and ii) rigid structure to suppress the non-radiative pathways from $T_{1}$. The preliminary computational conformer search revealed that $\mathbf{S i A z}$ can take 35 meta-stable conformers (Figure S18), which can take equatorial-equatorial (eqeq), equatorial-axial (eq-ax), or axial-axial (ax-ax) orientations. Further optimization of representative structures using density functional theory (DFT) calculations ( $\omega$ B97X-D/cc-pVDZ) revealed that eq-eq is always the dominant conformer in solutions (around 8.5 and $14.7 \mathrm{kcal} \mathrm{mol}^{-1}$ more stable than eq-ax and axax conformers, Figure 2). Excited state calculations revealed that there are substantial variations in energy alignment of $S_{1}$, $\mathrm{T}_{1}$, and $\mathrm{T}_{2}$ depending on the materials environments (Figure 2, for the details, see the Table S5-S10 in the Supporting Information). As expected from our previous works, ${ }^{23,25}$ the $S_{1}$ state has hybrid CT character in many solvents (methylcyclohexane: $\mathrm{MCH}$, toluene, and THF) (see the NTO pair of Figure 2). The $\mathrm{T}_{1}$ and $\mathrm{T}_{2}$ states in $\mathrm{MCH}$ and toluene are ${ }^{3} \mathrm{LE}_{\mathrm{A}}$ and ${ }^{3} \mathrm{CT}$ states, while the energy alignment is switched in a more polar solvent (THF) (Figure $2 \mathrm{~b}$ and $\mathrm{c}$ ). It should be noted that in less polar solvents such as $\mathrm{MCH}$ and toluene not only the energy alignment but also the magnitude relation of SOC matrix elements for $\mathrm{T}_{2}-\mathrm{S}_{0}\left(\right.$ ca. $\left.5.8 \mathrm{~cm}^{-1}\right), \mathrm{T}_{1}-\mathrm{S}_{1}\left(\right.$ ca. $\left.2.0-2.2 \mathrm{~cm}^{-1}\right)$, and $\mathrm{T}_{1}-\mathrm{S}_{0}$ (ca. $0.9 \mathrm{~cm}^{-1}$ ) transitions are ideal for designed RTP scenario (Figure 1c and Figure 2a). Furthermore, what was interesting is the significant stabilization of ${ }^{1} \mathrm{CT}$ energy in a polar environment (THF) would yield small $\Delta E_{\mathrm{ST}}$ enough for thermally activating RISC process for TADF. The set of predictions encouraged us to start this project to fine-tune the excited states energy alignment by molecular design and host materials. The designed material SiAz was successfully synthesized through a Pd-catalyzed double amination of dibrominated dibenzophenazine ${ }^{23}$ with a silicon-bridged diphenylamine (dihydrophenazasiline) in high yield (Equation S1). Thermogravimetric analysis revealed that $\mathbf{S i A z}$ is stable toward heating $(5 \mathrm{wt} \%$ loss temperature = $496{ }^{\circ} \mathrm{C}$ ), indicating the feasibility of fabrication of OLEDs devices by sublimation.

\section{Steady-state photophysics of $\mathrm{SiAz}$ in solutions and solid ma- trices.}

Initially, we investigated the ultraviolet-visible (UV-Vis) absorption and photoluminescence (PL) properties of SiAz. UVVis spectra (Figure S1a) in both non-polar and polar solvents displayed transitions below $480 \mathrm{~nm}$, similar to those seen in other $\mathrm{D}-\mathrm{A}-\mathrm{D}$ compounds containing dibenzo $[a, j]$ phenazine acceptor. $^{19,23,25}$ Concomitantly, steady-state emission spectra showed typical CT behaviour, where emissions bathochromically shifted as the polarity of solvents increases (Figure S1b). A degassed toluene solution of SiAz showed almost no difference in PL intensity from the aerated solution, indicating that there are little or none emissive pathways after the excursion tothe triplet state in solution (Figure 3a). Different results, however, were obtained in solid matrices, by removing oxygen from the sample of $1 \%$ of SiAz in Zeonex ${ }^{\circledR}$. The significant rise of the emission was observed, together with the new band appeari ing in the higher wavelength from the RTP process (Figure $3 b$ ). This concludes we have both emissions from singlet and triplet states in this system. ${ }^{9}$ As for the sample in DPEPO matrix, the increase of the emission is observed after degassing but the shape of the emission spectrum remains, which would suggest the triplet-driven emission is observed in this system and based on the literature this is a delayed fluorescence (DF) process 

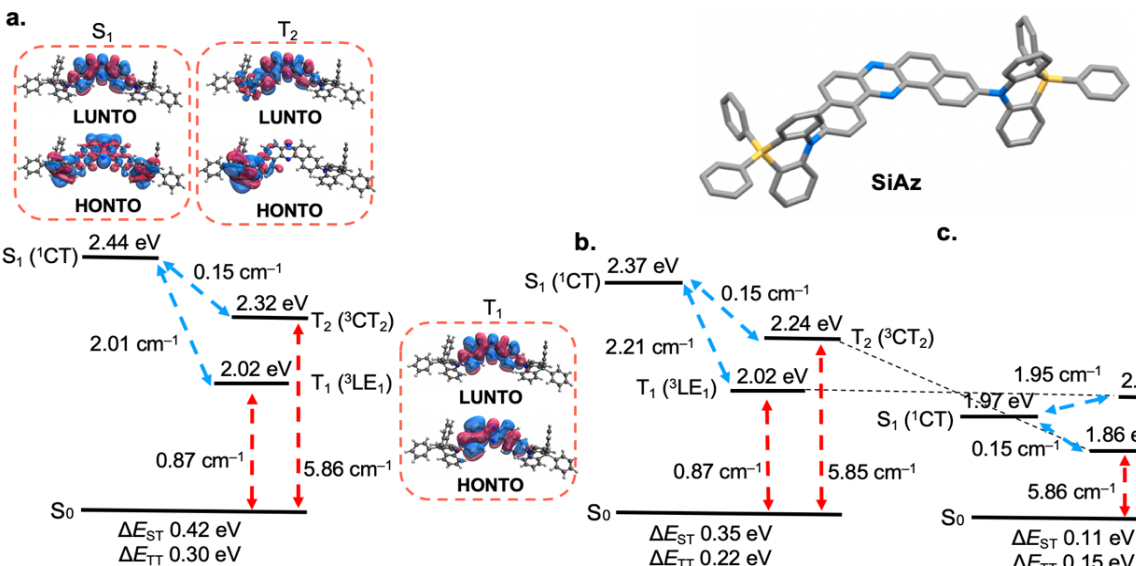

b.

c.

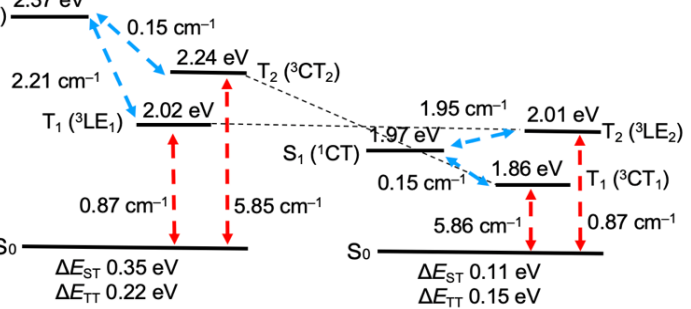

Figure 2. Summary of excited states energy alignment and NTO pairs of $\mathrm{S}_{1}, \mathrm{~T}_{1}$, and $\mathrm{T}_{2}$ states in (a) methylcyclohexane (MCH), (b) toluene, and (c) THF. The excited state energies were calculated with tuned $\omega$ PBE functional at the optimized $\mathrm{S}_{1}$ and $\mathrm{T}_{1}$ structures for the singlet and triplet manifolds respectively.
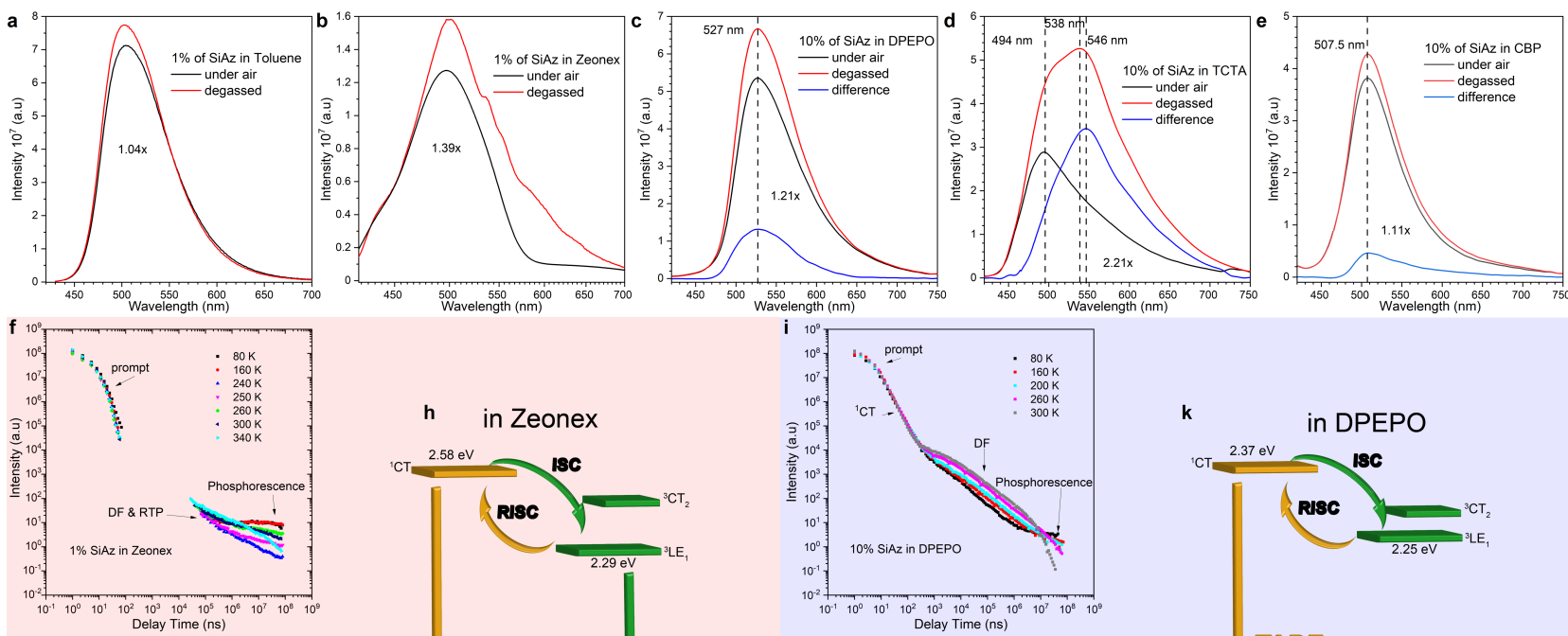

h
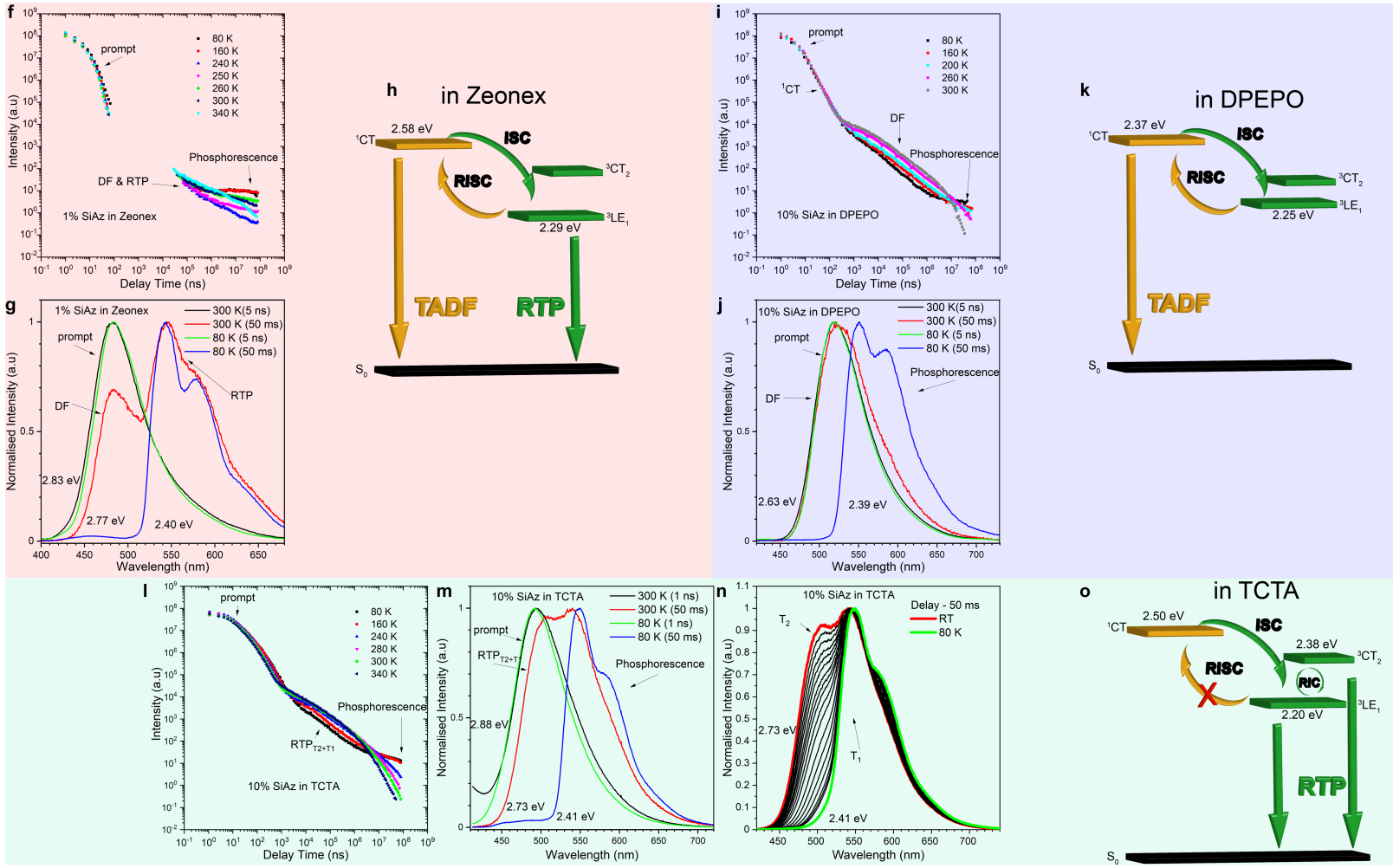

Figure 3. Steady-state spectra of (a) $1 \%$ w/w SiAz in Toluene, (b) 1\% w/w of SiAz in Zeonex ${ }^{\mathbb{R}}$, (c) $10 \%$ w/w of SiAz in DPEPO, (d) $10 \%$ w/w of SiAz in TCTA and (e) 10\% w/w of SiAz in CBP after (red lines) and before (black lines) removing oxygen from the system. The blue lines indicate the difference spectra of red and black lines. Photophysical properties of SiAz in host materials. (f) Intensity versus delay time at a different temperature, (g) emission spectra and (h) proposed mechanism of the emissions of $1 \% \mathrm{w} / \mathrm{w} \mathbf{S i A z}$ in Zeonex ${ }^{\circledR}$. (i) Intensity versus delay time at a different temperature, (j) emission spectra and (k) proposed mechanism of the emissions of 10\% w/w SiAz in DPEPO. (1) Intensity versus delay time at a different temperature, (m) emission spectra and (n) spectra at $80 \mathrm{~K}$ and long delays correspond to phosphorescence. Time-resolved spectra showing evolution of the $\mathrm{T}_{2}$ state emission of phosphorescence at $50 \mathrm{~ms}$ delay at different temperatures from $80 \mathrm{~K}$ up to $300 \mathrm{~K}$. (o) Proposed mechanism of the emissions of 10\% w/w SiAz in TCTA. Excited state energies are extracted from Table S3. 
(Figure 3c). ${ }^{16,19,20,21,23}$ The highest rise of the emission is observed in the TCTA matrix where there is 2.21 times rise of the emission with the appearance of a new band of longer wavelength, suggesting the RTP process (Figure $3 \mathrm{~d}$ ). ${ }^{9}$ The sample of SiAz in CBP also shows a small increase in the emission after oxygen removal, but the increase was limited to $1.1 \%$ of the original intensity (Figure 3e). Comparison of the emission spectra of SiAz in DPEPO, TCTA and CBP matrix before and after degassing revealed that only the $\mathbf{S i A z}$ in TCTA displays the distinct rise of a new band which is associated with RTP emission (Figure 3d). Otherwise, we are supposed to simply observe the rise of a similar CT emission band with those observed in CBP and DPEPO that would correspond to DF process rather than appearing of a new band (Figure 3d). The photoluminescence quantum yield of the degassed toluene solution was $22 \%$ (Figure $\mathrm{S} 2 \mathrm{~b}$ ), further suggesting that non-radiative deactivation pathways from the excited states play a major role in the decay kinetics of $\mathbf{S i A z}$ in solution. Intriguingly, aggregation-induced enhanced emission (AIEE) behaviour of $\mathbf{S i A z}$ was observed, when the ratio of water/THF blends increased (Figure S3).

\section{Time-resolved photophysics of $\mathrm{SiAz}$ in host matrices.}

$\mathbf{S i A z}$ affords complicated time-resolved photophysics, ${ }^{28}$ depending on the solid environments. The excited states energies extracted from the time-resolved photophysical spectroscopic measurements are summarized in Table S3. In order to elucidate the excited state dynamics in the solid-state, a film made by dispersing $\mathbf{S i A z}$ in Zeonex ${ }^{\circledR}$ matrix was spectroscopically analysed at temperatures ranging from 80 to $340 \mathrm{~K}$ in a time region between $1 \mathrm{~ns}$ and $79 \mathrm{~ms}$ (Figure $3 \mathrm{f}$ ). SiAz decays in two distinct time regions: one in the ns and the other in the ms region. Therefore, the decay profiles were almost devoid of emissive outputs in the $\mu$ s temporal time region, where delayed emission processes such as TADF, ${ }^{8} \mathrm{TTA}^{29}$ and triplet state emission ${ }^{[2]}$ are commonly observed. The emission detected in the ns region decays with a single exponential with an emission lifetime of $c a$. $4 \mathrm{~ns}$ at all temperatures.

The emission spectra acquired in the ns time region (0-36 ns) at $300 \mathrm{~K}$ showed Gaussian shapes with the identical onsets $(438 \mathrm{~nm}, 2.83 \mathrm{eV})$ and peaks $(481 \mathrm{~nm}, 2.58 \mathrm{eV})$, which are attributable to the emission from the ${ }^{1} \mathrm{CT}$ (Figure $3 \mathrm{~g}$ ). At first sight, $\mathbf{S i A z}$ appears to decay in the ms time region in a similar fashion at all temperatures (Figure $3 \mathrm{f}$ ). However, careful inspection of the spectra in the ms delay time revealed that a complicated competition between singlet and triplet excited states exists (Figure 3g). Firstly, in the ms time region, at temperatures below 240 K, SiAz showed a structured spectrum (onset $=516$ $\mathrm{nm}, 2.40 \mathrm{eV}$, blue line, Figure $3 \mathrm{~g}$ ), which is bathochromically shifted from the ${ }^{1} \mathrm{CT}$ emission observed in the prompt ns decay region (for the steady-state emission spectra at varied temperatures, see the Figure S6). The emission observed at low temperatures were identified as the phosphorescence irradiated from the locally excited triplet state of the acceptor $\left({ }^{3} \mathrm{LE}_{\mathrm{A}}\right)$, which was confirmed from the individual phosphorescence spectra of the acceptor. ${ }^{25}$ The $\Delta E_{\mathrm{ST}}\left({ }^{1} \mathrm{CT}-{ }^{3} \mathrm{LE}\right.$ gap $)$ calculated from the onset energies was larger than $370 \mathrm{meV}$, which corresponds to an energy gap proven to stymie the RISC process in many emissive materials. ${ }^{19-22}$ Additionally, the almost identical energy level of the ${ }^{3} \mathrm{LE}_{1}$ of $\mathrm{SiAz}$ with that of the acceptor $(2.40 \mathrm{eV})^{25}$ suggested that the D-A torsional angles are very close to $90^{\circ}$ (i.e., eq-orientation of donors against the acceptor), where the wavefunctions of the D and A moieties would be decoupled, and thereby manifesting the locally excited energetics of the acceptor. ${ }^{16}$ At temperatures above $240 \mathrm{~K}$, the millisecond delay time revealed dual emission comprising of ${ }^{1} \mathrm{CT}$ and triplet emissions (Figure $3 \mathrm{~g})$. The ${ }^{1} \mathrm{CT}$ emission was identified to be TADF by analysing the dependence of emission intensity on laser pulse fluency at $340 \mathrm{~K}$ (Figure S5). Since the TADF is only accessed at temperatures above $240 \mathrm{~K}$, the competition between the radiative decay from the ${ }^{3} \mathrm{LE}_{1}$ and the RISC from the ${ }^{3} \mathrm{LE}_{1}$ to the ${ }^{1} \mathrm{CT}$ was affirmed by the large energy barrier between the two excited states (Figure $3 \mathrm{~g}$ ). The RISC rate at $300 \mathrm{~K}$ esimated from eq 1 is $c a$. $10^{1}-10^{2} \mathrm{~s}^{-1}$, which ranges in similar decay rates with those of typical phosphorescence. ${ }^{30}$

$k_{R I S C}=\frac{I_{D F}}{I_{P F}} \frac{1}{\tau_{T A D F}}$

This justifies the presence of dual singlet and triplet emissions at $300 \mathrm{~K}$, allowing for a strong white emission at room temperature at $50 \mathrm{~ms}$ (Figure $3 \mathrm{~g}$ and Figure S6). While the observation of dual TADF/RTP is quite rare, it is not unprecedented in photophysical studies. ${ }^{31,32}$ Furthermore, Huang et al. demonstrated that the design principles for TADF and RTP are not mutually exclusive. ${ }^{31}$ Both mechanisms require the suppression of the decay from $\mathrm{S}_{1}$ to make $k_{\mathrm{ISC}}, k_{\mathrm{RISC}}$ (required for efficient TADF), and $k_{\text {phos }}$ (required only for RTP) competitive. They subsequently highlighted two important design principles: 1) angular molecular geometry within D-A-D scaffolds shifts $\mathrm{S}_{1}$ and $\mathrm{T}_{1}$ in closer proximity and facilitates ISC; 2 ) the presence of heteroatoms (even ones with a low atomic weight such as nitrogen $)^{33}$ enhances triplet formation. Similar considerations on molecular design were also addressed by Chen et. al. when designing phosphorescence compounds with fast ISC rates. ${ }^{34}$ In our case, SiAz satisfies Huang's design principles. Based on the $\Delta E_{\mathrm{ST}}(0.42 \mathrm{eV})$ obtained from theoretical calculations in $\mathrm{MCH}$, which can mimic the non-polar polymeric matrix environment (Figure 2 and Tables S5-10), and the behaviour of the emission, the emission observed in the Zeonex ${ }^{\circledR}$ matrix should be generated from the majority of eq-eq-type conformers. In Zeonex ${ }^{\circledR}$ matrix, experimental $\Delta E_{\mathrm{ST}}$ is on the edge value $(0.43$ $\mathrm{eV}$, Table S3) for efficient RISC, which is in good agreement with calculated one ( $\left.{ }^{\text {calc }} \Delta E_{\mathrm{ST}}=0.42 \mathrm{eV}\right)$, thereby reducing the RISC rate from ${ }^{3} \mathrm{LE}_{1}$ to ${ }^{1} \mathrm{CT}$ to show dual emission of TADF and RTP from ${ }^{3} \mathrm{LE}_{1}$ (Figure $\left.3 \mathrm{~h}\right)$. Given that ${ }^{\text {cal }} \Delta E_{\mathrm{TT}}(0.30 \mathrm{eV})$ is slightly smaller than ${ }^{\text {calc }} \Delta E_{\mathrm{ST}}(0.42 \mathrm{eV})$ and SOC matrix element between $T_{2}$ and $S_{0}$ is larger than that between $T_{1}$ and $S_{0}$ states, RTP from the $\mathrm{T}_{2}$ state might be involved in the emission (Figure $3 \mathrm{~h}$ ). This could be also experimentally reasonable, as three emission bands were observed in the steady-state spectra in Zeonex $^{\circledR}$ (Figure S6).

The white emission observed at room temperature in Zeonex ${ }^{\circledR}$ was not, however, observed in a polar matrix bis[2(diphenylphosphino)phenyl] ether oxide (DPEPO) (Figure 3i and j). In other words, RTP was not observed in this host. This can be explained by several accumulative factors. Firstly, the polarity of DPEPO, which contains phosphine oxide moieties, is much higher than that of the polymeric cyclic olefin matrix Zeonex $^{\circledR}$. Therefore, DPEPO stabilizes CT states over LE states, coaxing the ${ }^{1} \mathrm{CT}$ to be in closer resonance with the ${ }^{3} \mathrm{LE}$, which is evident from a bathochromic shift of the ${ }^{1} \mathrm{CT}$ emission (Figure $3 \mathrm{j}$ ). From the theoretical analysis, when compared to the case in MCH (approximate environment of Zeonex ${ }^{\circledR}$ host), THF (mimic of DPEPO host) allows for a decrease in the energy of 
${ }^{3} \mathrm{CT}_{2}$ to be lower than the ${ }^{3} \mathrm{LE}_{1}$ state, resulting in ${ }^{3} \mathrm{CT}_{1}$ and ${ }^{3} \mathrm{LE}_{2}$ (Figure 2 and Tables S5-10) close enough to form a resonance structure to feed an efficient RISC process. The triplet onset energies of the ${ }^{3} \mathrm{LE}_{1}$ in Zeonex ${ }^{\circledR}(2.40 \mathrm{eV})$ and DPEPO matrices $(2.39 \mathrm{eV})$ are very close (Table S3), implying that this ${ }^{3} \mathrm{LE}$ is less affected by host polarity than the ${ }^{1} \mathrm{CT}$ and ${ }^{3} \mathrm{CT}$ and also suggesting the same majority of eq-eq conformers irradiate the emission in the matrix. ${ }^{20}$ Both the prompt and delayed fluorescence decays displayed CT characteristics, which are bathochromically shifted from the emission in the Zeonex ${ }^{\circledR}$ matrix, with complicated kinetics (Figure 3i). This is highlighted upon inspection of the emission spectra associated with each delay region. As for SiAz in DPEPO matrix, a bathochromic shift in emission ranging from 460.9 to $482.5 \mathrm{~nm}(2.69-2.57 \mathrm{eV})$ at the time between 1 to 54 ns (Figure S7) was observed. This prompt emission from the singlet states does not decay with simple exponential kinetics, most likely due to the conformational heterogeneity of SiAz molecules in the solid rigid host. The delayed emission ( $>100 \mathrm{~ns}$ ) shows similar spectral heterogeneity with CT states closest to the triplet state decaying first $\left({ }^{1} \mathrm{CT}=497.4\right.$ $\mathrm{nm}$ at $2.8 \mu \mathrm{s}$ time delay), and molecules in conformations that do not promote such strong CT characteristics decay at later delay times $\left({ }^{1} \mathrm{CT}=476.7 \mathrm{~nm}\right.$ at $17 \mathrm{~ms}$ time delay). This diminished energy gap between ${ }^{3} \mathrm{LE}_{1}$ and ${ }^{1} \mathrm{CT}$, therefore, promotes the RISC process through faster decay (shorter lifetime) from the ${ }^{1} \mathrm{CT}$ state after accessing the triplet state. Therefore, both prompt and delayed time decays are not mono-exponential. In DPEPO, the observed significant red-shift of prompt emission from those in Zeonex ${ }^{\mathbb{R}}$ and TCTA is an indication of ${ }^{1} \mathrm{CT}$ emission, which is somewhat higher $(2.37 \mathrm{eV})$ than theoretical value (Figure 2c). Taking into consideration that the SOC matrix elements for the ${ }^{3} \mathrm{LE}$ and ${ }^{1} \mathrm{CT}$ states are always higher than those between ${ }^{3} \mathrm{CT}$ and ${ }^{1} \mathrm{CT}$ (Figure 2) and that phosphorescence spectra of SiAz in DPEPO matrix is similar to those in TCTA and Zeonex matrices (Figure S9), the RISC should occur from the $T_{1}$ state with ${ }^{3} \mathrm{LE}$ character, which then exclusively gives rise to TADF (Figure 3k).

The decay kinetics from SiAz appear even more complicated when impregnated into tris(4-carbazoyl-9ylphenyl)amine (TCTA) matrix. TCTA has lower polarity than DPEPO, which corresponds to a smaller bathochromic shift of the ${ }^{1} \mathrm{CT}_{1}$ band (Figure $3 \mathrm{~m}$ ). From inspection of the decay kinetics at different temperatures (Figure 31), we found that SiAz decays in three distinct time regions; one in the ns, $\mu$ s and the ms time region. While within the ns time regions, $\mathbf{S i A z}$ displayed depopulation from the ${ }^{1} \mathrm{CT}(2.88 \mathrm{eV}$, onset energy), with the first component being as prompt emission. The states in the $\mu \mathrm{s}$ and ms time region, however, are more ambiguous. The emission observed at $2.41 \mathrm{eV}$ (onset energy) in the ms delay times at low temperatures below $240 \mathrm{~K}$ was identified as phosphorescence from ${ }^{3} \mathrm{LE}_{1}$ state. At higher temperatures, up to $300 \mathrm{~K}$, this phosphorescence was still prevalent (displaying RTP in an OLED host matrix) and yet observed with the addition of a second hypsochromic peak which does not match the onset energy of the peak observed at prompt time scales (Figure $3 \mathrm{~m}$ ). To investigate this unique phenomenon, we conducted an additional photophysical analysis of $\mathbf{S i A z}$ in TCTA host, which revealed that long-lived emissive species (up to $50 \mathrm{~ms}$ ) are not a mixture of TADF and RTP but pure RTP from the $\mathrm{T}_{1}$ and $\mathrm{T}_{2}$ states (Figure $3 n$ ). From the analysis of the emission spectra of $\mathbf{S i A z}$ in TCTA at varied temperatures, only one new band peaked at 506 $\mathrm{nm}$ was appearing with a rise of the temperature (Figure 5). Since the two emission bands are quite close to each other, all excited energy would be transferred to the singlet state $\left({ }^{1} \mathrm{CT}\right)$ if this new band were TADF. But such a phenomenon was not observed here (Figure 3n), suggesting RTP processes. Also, from the theoretical analysis (toluene), one will notice that the singlet energy state $\left({ }^{1} \mathrm{CT}\right)$ is much higher than the triplet energy state $\left(\Delta E_{\mathrm{ST}}=0.35 \mathrm{eV}\right)$, and $\mathrm{T}_{1}$ and $\mathrm{T}_{2}$ energy gap $\left(\Delta E_{\mathrm{TT}}=0.22\right.$ $\mathrm{eV}$ ) is smaller than $\Delta E_{\mathrm{ST}}$. In addition, SOC matrix elements between $\mathrm{T}_{2}-\mathrm{S}_{0}\left(5.85 \mathrm{~cm}^{-1}\right)$ is much larger than that between $\mathrm{T}_{1}-$ $\mathrm{S}_{1}\left(2.21 \mathrm{~cm}^{-1}\right)$ (Figure 2). Moreover, the $\mathrm{T}_{2}$ state has $\mathrm{CT}$ and a slightly LE (hybrid CT) character (Figure 2), which would support the possibility to reduce the phosphorescence lifetime and observe much faster decay from ${ }^{3} \mathrm{CT}_{2}$ than from the phosphorescence from ${ }^{3} \mathrm{LE}_{1}$ state. The experimental energy difference between this state and $\mathrm{T}_{1}$ states $\left({ }^{\exp } \Delta E_{\mathrm{TT}}=0.20 \mathrm{eV}\right.$, Table S3) is smaller than that obtained from theoretical results (calc $\Delta E_{\mathrm{TT}}=$ $0.22 \mathrm{eV}$, Figure 2). This discrepancy would be rationalized by i) a larger dielectric constant of TCTA than toluene and ii) lower $\mathrm{T}_{2}$ energy at the $\mathrm{T}_{2}$ geometry than that at the $\mathrm{T}_{1}$ geometry (vide infra). In TCTA, similar to the RISC process in the TADF mechanism, SOC mixes the different spin states and dissolves the forbidden singlet-triplet transitions. The mixed CT character of $\mathrm{T}_{2}$ state allows for the much faster photo-irradiation process than from $\mathrm{T}_{1}$ (Figure 3o), which is supported by the large difference in the calculated SOC matrix elements in toluene (Figure $2 \mathrm{~b}$ ). Hence, the real excited "triplet" state is not a pure triplet state but has some singlet character, and therefore transition to the $\mathrm{S}_{0}$ is more probable even at room temperature. Therefore, fast radiative decay process from the $T_{2}$ state is observed at such long times, it would be mainly because the $T_{2}$ state is fed from long-lived $\mathrm{T}_{1}$ state through thermally activated reverse internal conversion (RIC, Figure 3o). Through the mechanism, so as long as the $\mathrm{T}_{1}$ state allows for RIC process, the short-lived $\mathrm{T}_{2}$ emission will be observed (Figure 3o).

\section{OLED fabrication and characterization.}

To investigate whether this material works in OLED devices, the electrochemical behaviour of SiAz was analyzed. The ionization potential (IP) $(5.85 \mathrm{eV})$ and electron affinity (EA) (3.49 $\mathrm{eV}$ ) were determined with cyclic voltammetry (Figure S14), ${ }^{35-}$ ${ }^{37}$ and they were found at similar potentials to the reported DA-D emitters containing a dibenzo $[a, j]$ phenazine acceptor. ${ }^{23,25}$ The behaviour of SiAz in 3 hosts, CBP [4,4'-bis( $N$-carbazolyl)1,1'-biphenyl], DPEPO, and TCTA, was then determined in OLED devices. ${ }^{[38,39]}$ The 3 OLED devices were fabricated and analysed in the following configurations: Device 1 - ITO/NPB $(40 \mathrm{~nm}) / \mathrm{TSBPA}(10 \mathrm{~nm}) / 10 \% \mathrm{SiAz}$ in CBP $(30 \mathrm{~nm}) / \mathrm{TPBi}$ [2,2',2"-(1,3,5-benzinetriyl)-tris(1-phenyl-1- $H$-benzimidazole)] $(60 \mathrm{~nm}) / \mathrm{LiF}(1 \mathrm{~nm}) / \mathrm{Al}(100 \mathrm{~nm})$; Device 2 - ITO/HATCN $(10 \mathrm{~nm}) / \mathrm{NPB}(30 \mathrm{~nm}) / \mathrm{mCP}(10 \mathrm{~nm}) / 10 \% \mathrm{SiAz}$ in DPEPO $(20 \mathrm{~nm}) / \mathrm{TPBi}(60 \mathrm{~nm}) / \mathrm{LiF}(1 \mathrm{~nm}) / \mathrm{Al}(100 \mathrm{~nm})$; Device 3 ITO/NPB (40 nm)/TAPC [4,4'-cyclohexylidenebis( $N, N$-bis(4methylphenyl)benzenamine) $](10 \mathrm{~nm}) / 10 \% \mathbf{S i A z}$ in TCTA (30 $\mathrm{nm}) / \mathrm{TPBi}(60 \mathrm{~nm}) / \mathrm{LiF}(1 \mathrm{~nm}) / \mathrm{Al}(100 \mathrm{~nm})$ (Figure 4). The characteristics of the OLED devices revealed a significant increase of OLED efficiency depending on the host used. The device based on CBP was found be the most efficient $(5.27 \%$ EQE), but it showed substantial roll-off and luminance up to $\left(17,240 \mathrm{~cd} / \mathrm{m}^{2}\right)$ in comparison to the devices fabricated with other hosts, subsequently making DPEPO and TCTA better hosts. The DPEPO host also shifted the EL of SiAz to the lower energies and gave a boost in the form of TADF enhancement up to $5.32 \% \mathrm{EQE}$ and $17,105 \mathrm{~cd} / \mathrm{m}^{2}$ luminance (Figure $4 \mathrm{~b}$ ). 

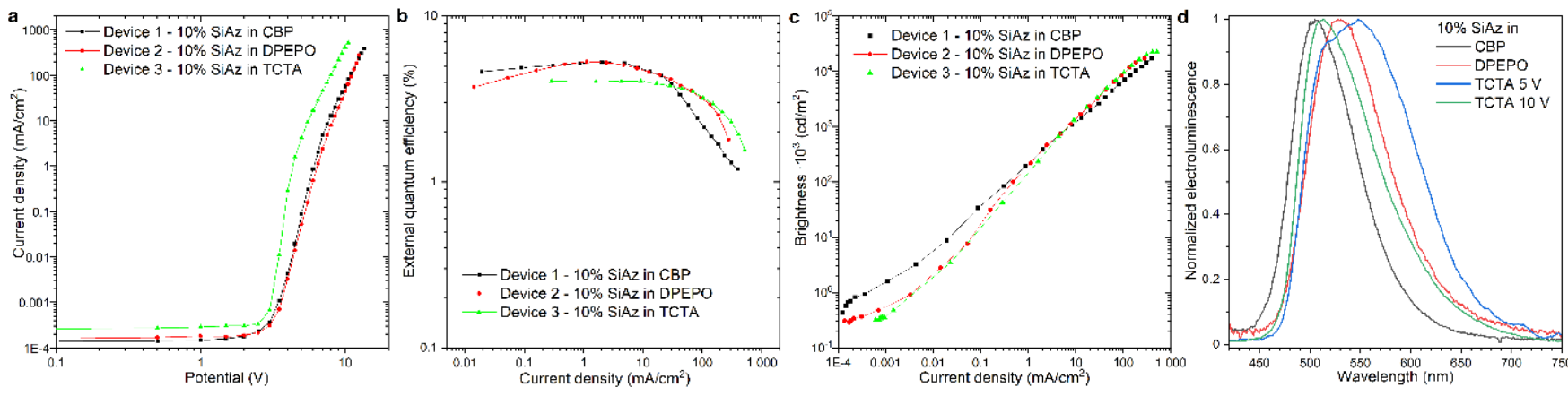

.
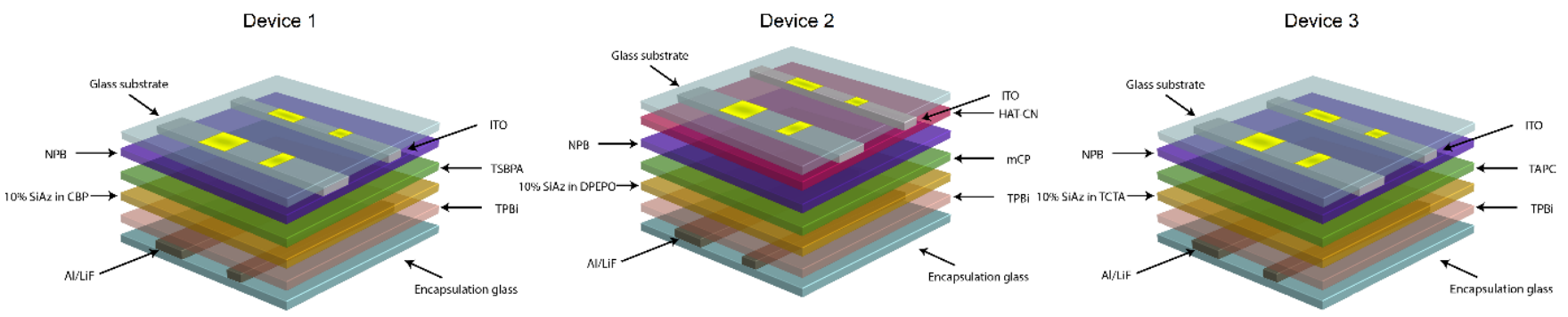

Figure 4. The characteristics of the unencapsulated OLED devices fabricated with different structures measured at room temperature in glovebox conditions. (a) Current density-bias characteristics. (b) EQE-current density characteristics. (c) Luminance-current density characteristics. (d) Electroluminescence spectra. (e) Device structures.

As for the TCTA-based device, the current response and the efficiency behaved similarly to other devices, resulting in $4.06 \% \mathrm{EQE}$ and $22,561 \mathrm{~cd} / \mathrm{m}^{2}$ luminance (Figure 4). However, unique to all other host materials was the observation of a change in emission with increasing voltage. Devices based on the TCTA host showed two emissions, at lower potential $<7 \mathrm{~V}$ and at higher potential $>9 \mathrm{~V}$ and a mixture in between these voltages (Figure 4d). This voltage-dependent emission is reversible, and therefore, we concluded that this behaviour is not caused by the degradation of the device. The emission in a lower voltage gave $4.06 \% \mathrm{EQE}$ and up to $2,000 \mathrm{~cd} / \mathrm{m}^{2}$ luminance, whereas the brighter one in a higher voltage exhibited much lower efficiency ( $c a .2 \% \mathrm{EQE}$ ) with much higher luminance (above $20,000 \mathrm{~cd} / \mathrm{m}^{2}$ ). This clearly shows that there are 2 different processes involved in the same device. The phosphorescence from the mixed ${ }^{3} \mathrm{LE}_{1}$ and ${ }^{3} \mathrm{CT}_{2}$ states matches the EL from the device at a low voltage, while the EL observed at a higher potential matches the majority of emission from ${ }^{3} \mathrm{CT}_{2}$ states as the population from ${ }^{3} \mathrm{CT}_{2}$ increased (Figure $4 \mathrm{~d}$ ). When all devices are compared, there is a correlation between $\Delta E_{\mathrm{ST}}$ and device efficiency. The devices with the smaller gap ( $c a .0 .3$ $\mathrm{eV}$ ) showed efficiency around 5\% (CBP, DPEPO, and TCTAbased devices), where the gap around $0.4 \mathrm{eV}$ produced devices with only $2 \%$ efficiency $(\mathrm{mCP})$.

\section{Conclusion}

In conclusion, we have successfully developed a unique purely organic emitter $\mathbf{S i A z}$ that could revolutionize the approach in designing novel OLED materials. The introduction of less-electron-donating, rigid, and sterically hindered donors into the acceptor suppressed the rotation and vibration around the $\mathrm{D}-\mathrm{A}$ units, which turned-on RTP emission. Depending on the hosts in which emitter was impregnated, TADF, RTP, or both emission channels were boosted. This characteristic offers unique opportunities for tailoring emission properties of OLED devices by choosing hosts and applied voltages. Moreover, white emission within a single organic emitter was achieved by joining RTP and TADF emission in Zeonex ${ }^{\circledR}$ matrix. Most importantly, not only were we able to prove the significant involvement of RTP in OLEDs emission from photophysical analyses but also we realized the most efficient heavy atom-free RTP-based OLED device (4.06\%) to date.

\section{Experimental Section}

Synthesis of SiAz. The synthetic procedures and spectroscopic characterization of SiAz are described in Section 2 (SI-2) of the Supplementary Information.

Photophysics. All steady-state absorption spectroscopy was performed on a UV-3600 Shimadzu spectrophotometer. Steady-state photoluminescence spectra were performed on a Jobin Yvon Horiba Fluorolog 3. The photoluminescence quantum yield (PLQY) of SiAz in toluene solvent was determined using the reference method against 9,10-diphenylanthracene (DPA) $\left(\varphi_{\mathrm{f}}=0.90\right.$ in cyclohexane $)$. Phosphorescence, prompt fluorescence (PF), and delayed fluorescence (DF) spectra and decays were recorded using nanosecond gated luminescence and lifetime measurements (from $\sim 500$ ps to $100 \mathrm{~ms}$ ) using either third harmonics of high energy pulsed Nd:YAG laser emitting at $355 \mathrm{~nm}$ (EKSPLA) or a $\mathrm{N}_{2}$ laser emitting at $337 \mathrm{~nm}^{34}$ Emission was focused onto a spectrograph and detected on a sensitive gated iCCD camera (Stanford Computer Optics) having a sub-nanosecond resolution. Temperature-dependent experiments were conducted using a continuous flow liquid nitrogen cryostat (Janis Research) under a nitrogen atmosphere.

Devices. OLEDs have been fabricated on pre-cleaned, patterned indium-tin-oxide (ITO) coated glass substrates with a sheet 
resistance of $20 \Omega / \mathrm{sq}$ and ITO thickness of $100 \mathrm{~nm}$. All small molecules and cathode layers were thermally evaporated in Kurt J. Lesker SuperSpectros 200 evaporation system under pressure of $10^{-7} \mathrm{mbar}$ without breaking the vacuum. The sizes of pixels were $4 \mathrm{~mm}^{2}, 8 \mathrm{~mm}^{2}$ and $16 \mathrm{~mm}^{2}$. Each emitting layer has been formed by co-deposition of dopant and host at the specific rate to obtain $10 \%$ content of the emitter. The characteristics of the devices were recorded using a 6-inch integrating sphere (Labsphere) inside the glovebox connected to a Source Meter Unit and Ocean Optics USB4000 spectrometer. ${ }^{38,39}$ NPB ( $N, N^{\prime}$-di(1-naphthyl)- $N, N^{\prime}$-diphenyl-(1,1'-biphenyl)-4,4'-diamine), TCBPA (4,4'-(Diphenylmethylene)bis( $N, N$-diphenylaniline)), CzSi (9-(4-tert-butylphenyl)-3,6-bis(triphenylsilyl)$9 H$-carbazole), TAPC $\left(4,4^{\prime}\right.$-Cyclohexylidenebis $[N, N$-bis(4methylphenyl)benzenamine]), TPBi $\left(2,2^{\prime}, 2^{\prime \prime}-(1,3,5\right.$-Benzinetriyl)-tris(1-phenyl-1- $H$-benzimidazole)), TCTA (Tris(4carbazoyl-9-ylphenyl)amine), $\quad \mathrm{mCP} \quad$ (1,3-Bis(carbazol-9yl)benzene), CBP (4,4'-bis( $N$-carbazolyl)-1,1'-biphenyl), DPEPO (Bis[2-(diphenylphosphino)phenyl] ether oxide) were sublimed before use.

Electrochemistry. All cyclic voltammetry measurements were performed at room temperature with a scan rate of $100 \mathrm{mV} \mathrm{s}^{-1}$. Ionization potential (IP) and electron affinity (EA) were calculated from oxidation $\left(E_{\mathrm{ox}}\right)$ and reduction $\left(E_{\mathrm{red}}\right)$ potentials, respectively, using following equations: $\mathrm{IP}(\mathrm{eV})=E_{\mathrm{ox}} / \mathrm{V}+5.1$, $\mathrm{EA}(\mathrm{eV})=E_{\text {red }} / \mathrm{V}+5.1 .{ }^{35-37}$

Calculations. A conformation search of $\mathrm{SiAz}$ was conducted using Schrödinger Materials Science Suite (Version 2.5). DFT calculations were done using QChem 5.0 software package. ${ }^{40}$ The $\omega \mathrm{B} 97 \mathrm{X}-\mathrm{D}$ functional was used for geometry optimizations and optimally tuned $\omega$ PBE for calculations of excited state levels. Non-equilibrium PCM models was used to capture solvation effects. Further details of calculations can be found in the Supplementary Information.

\section{ASSOCIATED CONTENT}

Supporting Information.

The Supporting Information is available free of charge via the Internet at http://pubs.acs.org.

Experimental procedures for the syntheses of materials, spectroscopic data of new compounds, steady-state UV-vis and PL spectra of $\mathbf{S i A z}$ in solutions, Aggregation-induced enhanced emission characteristics of $\mathbf{S i A z}$, phosphorescence spectra of the donor in solid matrix, additional time-resolved and temperature-dependent PL spectra of SiAz in matrices, theoretical details and the list of excited energies of $\mathbf{S i A z}$ in solid matrices, cyclic voltammetry of SiAz, thermogravimetric analysis (TGA) profiles, and copies of the ${ }^{1} \mathrm{H}$ and ${ }^{13} \mathrm{C}$ NMR spectra of new compounds (PDF)

\section{AUTHOR INFORMATION}

\section{Corresponding Author}

*pdes@dtu.dk

*takeda@chem.eng.osaka-u.ac.jp

* przemyslaw.data@polsl.pl

Notes

The authors declare no competing financial interest.
P.D. acknowledges the support received from the First Team program of the Foundation for Polish Science co-financed by the European Union under the European Regional Development Fund (project number: POIR.04.04.00-00-4668/17-00). YT and PD acknowledge the EU's Horizon 2020 for funding the OCTA project under grant agreement No 778158. Y.T. acknowledges a Grant-inAid for Scientific Research on Innovative Areas " $\pi$-System Figuration: Control of Electron and Structural Dynamism for Innovative Functions" (JSPS KAKENHI Grant Number JP15H00997 \& JP17H05155) and "Aquatic Functional Materials: Creation of New Materials Science for Environment-Friendly and Active Functions" (JSPS KAKENHI Grant Number JP19H05716) from the MEXT (Ministry of Education, Culture, Science and Technology, Japan), and the Research Grant from the Japan Prize Foundation, and the Continuation Grants for Young Researchers from the Asahi Glass Foundation, and the Research Grant in the Natural Science from the Mitsubishi Foundation.

\section{REFERENCES}

(1) Bernanose, A.; Comte, M.; Vouaux, P. Sur Un Nouveau Mode D'émission Lumineuse Chez Certains Composés Organiques. $J$. Chim. Phys. 1953, 50, 64-68.

(2) Sasabe, H.; Kido, J. Recent Progress in Phosphorescent Organic Light-Emitting Devices. Eur. J. Org. Chem. 2013, 2013, 76537663.

(3) Organic Light-Emitting Diodes (OLEDs) Materials, Devices and Applications; Buckley, A., Ed.; v

(4) Im, Y.; Kim, M.; Cho, Y. J.; Seo, J.-A; Yook, K. S.; Lee, J. Y. Molecular Design Strategy of Organic Thermally Activated Delayed Fluorescence Emitters. Chem. Mater. 2017, 29, 1946-1963.

(5) Yang, X.; Xu, X.; Zhou, G. Recent advances of the emitters for high performance deep-blue organic light-emitting diodes. $J$. Mater. Chem. C 2015, 3, 913-944.

(6) Kalinowski, J. Organic Light Emitting Diodes: Principles, Characteristics and Processes; Marcel Dekker: New York, 2005.

(7) Baldo, M. A.; O’Brian, D. F.; You, Y.; Shoustikov, A.; Sibley, S.; Thompson, M. E.; Forrest, S. R. Highly efficient phosphorescent emission from organic electroluminescent devices. Nature 1998, 395, 151-154.

(8) Dias, F. B.; Penfold, T. J.; Monkman, A. P. Photophysics of thermally activated delayed fluorescence molecules. Methods Appl. Fluoresc. 2017, 5, 012001/1-25.

(9) Nobuyasu, R. S.; Ren, Z.; Griffiths, G. C.; Batsanov, A. S.; Data, P.; Yan, S.; Monkman, A. P.; Bryce, M. R.; Dias, F. B. Rational Design of TADF Polymers Using a Donor-Acceptor Monomer with Enhanced TADF Efficiency Induced by the Energy Alignment of Charge Transfer and Local Triplet Excited States. $A d v$. Opt. Mater. 2016, 4, 597-607.

(10) Jankus, V.; Data, P.; Graves, D.; McGuinness, C.; Santos, J.; Bryce, M. R.; Dias, F. B.; Monkman, A. P. Highly Efficient TADF OLEDs: How the Emitter-Host Interaction Controls Both the Excited State Species and Electrical Properties of the Devices to Achieve Near $100 \%$ Triplet Harvesting and High Efficiency. Adv. Funct. Mater. 2014, 24, 6178-6186.

(11) Uoyama, H.; Goushi, K.; Shizu, K.; Nomura, H.; Adachi, C. Highly efficient organic light-emitting diodes from delayed fluorescence. Nature 2012, 492, 234-238.

(12) Hirata, S.; Totani, K.; Zhang, J.; Yamashita, T.; Kaji, H.; Marder, S. R.; Watanabe, T.; Adachi, C. Efficient Persistent Room Temperature Phosphorescence in Organic Amorphous Materials under Ambient Conditions. Adv. Funct. Mater. 2013, 23, 33833397.

(13) Chaudhuri, D.; Sigmund, E.; Meyer, A.; Röck, L.; Klemm, P.; Lautenschlager, S.; Schmid, A.; Yost, S. R.; Van Voorhis, T.; Bange, S.; Höger, S.; Lupton, J. M. Metal-Free OLED Triplet Emitters by Side-Stepping Kasha's Rule. Angew. Chem., Int. Ed. 2013, 52, 13449-13452.

\section{ACKNOWLEDGMENT}


(14) Data, P.; Takeda, Y. Recent Advancements in and the Future of Organic Emitters: TADF- and RTP-Active Multifunctional Organic Materials. Chem. Asian J. 2019, 14, 1613-1636.

(15) Reineke, S. Phosphorescence meets its match. Nat. Photonics 2014, 8, 269-270.

(16) Dias, F. B.; Bourdakos, K. N.; Jankus, V.; Moss, K. C.; Kamtekar, K. T.; Bhalla, V.; Santos, J.; Bryce, M. R.; Monkman, A. P. Triplet Harvesting with $100 \%$ Efficiency by Way of Thermally Activated Delayed Fluorescence in Charge Transfer OLED Emitters. Adv. Mater. 2013, 25, 3707-3714.

(17) Ward, J. S.; Nobuyasu, R. S.; Batsanov, A. S.; Data, P.; Monkman, A. P.; Dias, F. B.; Bryce, M. R. The interplay of thermally activated delayed fluorescence (TADF) and room temperature organic phosphorescence in sterically-constrained donor-acceptor charge-transfer molecules. Chem. Commun. 2016, 52, 2612 2615.

(18) de Silva, P.; Kim, C. A.; Zhu, T.; Van Voorhis, T. Extracting Design Principles for Efficient Thermally Activated Delayed Fluorescence (TADF) from a Simple Four-State Model. Chem. Mater. 2019, 31, 6995-7006.

(19) Takeda, Y.; Kaihara, T.; Okazaki, M.; Higginbotham, H.; Data, P.; Tohnai, N.; Minakata, S. Conformationally-flexible and moderately electron-donating units-installed $\mathrm{D}-\mathrm{A}-\mathrm{D}$ triad enabling multicolor-changing mechanochromic luminescence, TADF and room-temperature phosphorescence. Chem. Commun. 2018, 54, $6847-6850$.

(20) dos Santos, P. S.; Ward, J. S.; Bryce, M. R.; Monkman, A. P. Using Guest-Host Interactions To Optimize the Efficiency of TADF OLEDs. J. Phys. Chem. Lett. 2016, 7, 3341-3346.

(21) Data, P.; Okazaki, M.; Minakata, S.; Takeda, Y. Thermally activated delayed fluorescence $v s$. room temperature phosphorescence by conformation control of organic single molecules. $J$. Mat. Chem. C 2019, 7, 6616-6621.

(22) de Silva, P. Inverted Singlet-Triplet Gaps and Their Relevance to Thermally Activated Delayed Fluorescence. J. Phys. Chem. Lett. 2019, 10, 5674-5679.

(23) Okazaki, M.; Takeda, Y.; Data, P.; Pander, P.; Higginbotham, H.; Monkman, A. P.; Minakata, S. Thermally activated delayed fluorescent phenothiazine-dibenzo[ $[a, j]$ phenazine-phenothiazine triads exhibiting tricolor-changing mechanochromic luminescence. Chem. Sci. 2017, 8, 2677-2686.

(24) He, Z.; Zhao, W.; Lam, J. W. Y.; Peng, Q.; Ma, H.; Liang, G.; Shuai, Z.; Tang, B. Z. White light emission from a single organic molecule with dual phosphorescence at room temperature. Nat. Commun. 2017, 4, 416/1-8.

(25) Data, P.; Pander, P.; Okazaki, M.; Takeda, Y.; Minakata, S.; Monkman, A. P. Dibenzo[a,j]phenazine-Cored Donor-Acceptor-Donor Compounds as Green-to-Red/NIR Thermally Activated Delayed Fluorescence Organic Light Emitters. Angew. Chem., Int. Ed. 2016, 55, 5739-5744.

(26) Aydemir, M.; Xu, S.; Chen, C.; Bryce, M. R.; Chi, Z.; Monkman, A. P. Photophysics of an Asymmetric Donor-Acceptor-Donor' TADF Molecule and Reinterpretation of Aggregation-Induced TADF Emission in These Materials. J. Phys. Chem. C 2017, 121, 17764-17772.
(27) Wu, Q.; Zhang, T.; Peng, Q.; Wang, D.; Shuai, Z. Aggregation induced blue-shifted emission - the molecular picture from a QM/MM study. Phys. Chem. Chem. Phys. 2014, 16, 5545-5552.

(28) Pander, P.; Data, P.; Dias, F. B. Time-resolved Photophysical Characterization of Triplet-harvesting Organic Compounds at an Oxygen-free Environment Using an iCCD Camera. J. Vis. Exp. 2018, 140, e56614/1-9.

(29) Luo, Y.; Aziz, H. Correlation Between Triplet-Triplet Annihilation and Electroluminescence Efficiency in Doped Fluorescent Organic Light-Emitting Devices. Adv. Funct. Mater. 2010, 20, $1285-1293$.

(39) Köhler, A.; Bässler, H. Triplet states in organic semiconductors. Mater. Sci. Eng. R 2009, 66, 71-109.

(40) Huang, R.; Avó, J.; Northey, T.; Chaning-Pearce, E.; dos Santos, P. L.; Ward, J. S.; Data, P.; Etherington, M. K.; Fox, M. A.; Penfold, T. J.; Berberan-Santos, M. N.; Lima, J. C.; Bryce, M. R.; Dias, F. B. The contributions of molecular vibrations and higher triplet levels to the intersystem crossing mechanism in metal-free organic emitters. J. Mater. Chem. C 2017, 5, 6269-6280.

(41) Hofbeck, T.; Monkowius, U.; Yersin, H. Highly Efficient Luminescence of $\mathrm{Cu}(\mathrm{I})$ Compounds: Thermally Activated Delayed Fluorescence Combined with Short-Lived Phosphorescence. $J$. Am. Chem. Soc. 2015, 137, 399-404.

(42) Reineke, S.; Baldo, M. Room temperature triplet state spectroscopy of organic semiconductors. Sci. Rep. 2014, 4, 3797/1-8.

(43) Chen, X.; Xu, C.; Wang, T.; Zhou, C.; Du, J.; Wang, Z.; Xu, H.; Xie, T.; Bi, G.; Jiang, J.; Zhang, X.; Demas, J. N.; Trindle, C. O.; Luo, Y.; Zhang, G. Versatile Room-Temperature-Phosphorescent Materials Prepared from N-Substituted Naphthalimides: Emission Enhancement and Chemical Conjugation. Angew. Chem., Int. Ed. 2016, 55, 9872-9876.

(44) Cardona, M.; Li, W.; Kaifer, A. E.; Stockdale, D.; Bazan, G. C. Electrochemical Considerations for Determining Absolute Frontier Orbital Energy Levels of Conjugated Polymers for Solar Cell Applications. Adv. Mater. 2011, 23, 2367-2371.

(45) Data, P.; Motyka, R.; Lapkowski, M.; Suwinski, J.; Monkman, A. P. Spectroelectrochemical Analysis of Charge Carriers as a Way of Improving Poly( $p$-phenylene)-Based Electrochromic Windows. J. Phys. Chem. C 2015, 119, 20188-20200.

(46) Pluczyk, S.; Vasyilieva, M.; Data, P. Using Cyclic Voltammetry, UV-Vis-NIR, and EPR Spectroelectrochemistry to Analyze Organic Compounds. J. Vis. Exp. 2018, 140, e56656/1-13.

(47) de Sá Pereira, D.; Data, P.; Monkman, A. P. Methods of Analysis of Organic Light Emitting Diodes. Disp. Imaging 2017, 2, 323 337.

(48) de Sa Pereira, D.; Monkman, A. P.; Data, P. J. Vis. Exp. 2018, 140, e56593/1-6.

(49) Shao, Y.; Gan, Z.; Epifanovsky, E.; Gilbert, A. T. B.; Wormit, M.; Kussmann, J.; Lange, A. W.; Behn, A.; Deng, J.; Feng, X. and et al. Advances in molecular quantum chemistry contained in the Q-Chem 4 program package. Mol. Phys. 2015, 113, 184 215. 


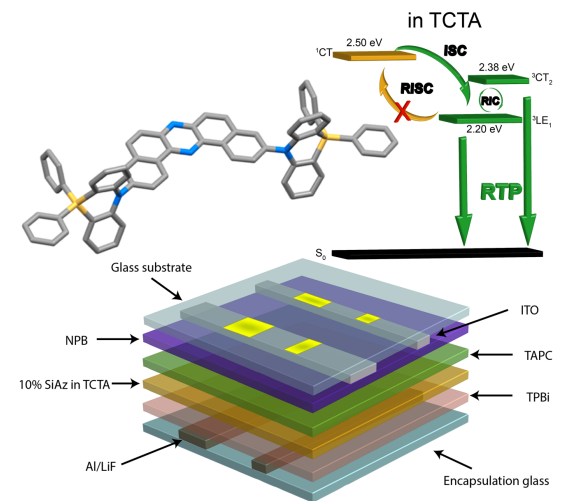

